\title{
CUANDO MI CENTRO EDUCATIVO ES UNA CÁRCEL Y MIS ESTUDIANTES SON PRIVADOS DE LIBERTAD: UNA RECONSTRUCCIÓN DE LA PRÁCTICA DOCENTE EN EL CENTRO DE FORMACIÓN JUVENIL ZURQUI
}

\author{
Maura N. Espinoza Rostrán* \\ Ayrieth Granados Carrillo**
}

El trabajo que a continuación se expone, es parte de una investigación que se elaboró para optar por el grado de Licenciatura en Ciencias de la Educación, con énfasis en Didáctica de los Estudios Sociales. Aquel lleva como objetivo, caracterizar la práctica pedagógica en el Centro de Formación Juvenil Zurquí, una cárcel de menores ubicada en San Luis de Santo Domingo, Heredia, mediante una investigación cualitativa. Es decir, estudiar las condiciones inherentes al aprendizaje en dicho espacio, el qué, el cómo y el para qué de la educación, los sujetos cognoscentes, las características del proceso de aprendizaje dentro de un sistema carcelario cerrado, y finalmente, ¿cuáles condiciones caracterizan y diferencian a este centro de otras instituciones educativas?.

El valor agregado de este trabajo radica en que, al reconstruir la práctica docente y rehacerla en forma teórica, aplicando un enfoque cualitativo de investigación, el profesor se convierte en un investigador de su propia práctica educativa, se involucra en la investigación curricular y, en la misma dinámica institucional; ello implica una interacción activa entre el docente investigadory sus informantes.
This article is part of a broader research which was developed as a requirement to obtain the degree of licenciate in Education Sciences with emphasis in Social Studies Didactis. The study had the aim of characterizing pedagogical practices at the Centro de Formación Juvenil Zurqui, a youth jail located in San Luis de Santo Domingo de Heredia, by means of a qualitative research. In other words, its objective was to study the specific learning conditions in that environment, the what, how and why of education, the learning individuals, the leaming process characteristics within a closed penitentiary system, and finally, it questions the conditions that characterize and differentiate this center from other education centers.

The aggregated value of this work is that in rebuilding the teaching practice and remaking it theoretically, applying a qualitative research approach, the teacher becomes a researcher of his own education practice; besides, he or she gets involved in curriculum research and institutional dynamics; that implies an active interaction between the researching teacher and his (her) informants.

* Maura Nelia Espinoza Rostrán, Licenciada en Ciencias de la Educación con énfasis en Didáctica de los Estudios Sociales, Universidad Nacional; Bachiller en Historia, Bachiller en la Enseñanza de los Estudios Sociales, Universidad Nacional; Diplomada en I y II Ciclo, UNED, cursa el Programa de Maestría en Planificación Curricular, U.C.R. Actualmente labora en el Colegio de San Luis Gonzaga. Cartago.

** Ayrieth Granados Carrillo, Licenciada en Ciencias de la Educación con énfasis en Didáctica de los Estudios Sociales, Universidad Nacional; Bachiller en Historia y en la Enseñanza de los Estudios Sociales, Universidad Nacional. Actualmente labora para el Ministerio de Justicia en calidad de docente, en el Centro de Formación Juvenil Zurquí, Heredia 


\section{Los motivos de esta investigación.}

El salón de clases es un espacio vital y complejo, que se encuentra mediatizado por intereses, emociones, sentimientos, visiones del mundo y del ser humano, valores y hasta prejuicios. Mejorar la educación implica: estudiar y reconstruir la práctica pedagógica, por medio de un proceso de análisis reflexivo a partir de la realidad "aulica" misma. Si el aprendizaje en condiciones formales es complejo, lo es aún más, cuando el centro educativo se encuentra inmerso dentro de un centro cerrado de atención a la población penal juvenil.

Esta institución corresponde a una cárcel juvenil ubicada en San Luis de Santo Domingo, Heredia y en él funciona una escuela llamada "Corazón de María”. Aquel centro penal surge como una respuesta más del Estado Costarricense, al fenómeno de la delincuencia juvenil y a la misma impunidad que se evidencia en delitos de suma gravedad tales como, homicidios, violaciones o agresiones. Al crearse la Ley de Justicia Penal Juvenil en 1996, (UNICEF, 2000) y contemplar regulaciones importantes en materia educativa, que deben cumplirse en los centros cerrados de atención a las personas infractoras menores de edad, el Centro de Formación Juvenil Zurquí se ha convertido en un modelo para América Latina, ya que propicia una serie de condiciones para el desarrollo del muchacho, incluida una educación dirigida a su reinserción en la sociedad, sobre la base del Programa de Alfabetización y Educación General Básica Abierta y Diversificada.

El resultado legal de cometer un delito es solamente la pérdida de la libertad. Los otros derechos inherentes a todas las personas menores de edad, deben ser garantizados por el estado, a pesar de su proceso de prisionalización; uno de ellos es la educación. Así consta en el artículo 77 de nuestra Constitución Política (Constitución Política, 1995). Además de los documentos jurídicos nacionales, existe una serie de instrumentos jurídicos internacionales de acatamiento obligatorio, que contemplan disposiciones en materia educativa que deben ponerse en práctica en nuestro país, entre ellas las Normas de Beijing y Reglas de las Naciones Unidas para la protección de los Menores Privados de libertad (Rojas, 1996).

\section{Los constructos teóricos y los referentes metodológicos}

La Educación busca desarrollar en forma integrada las facultades físicas, intelectuales y morales del niño, a partir de las características del educando y su medio físico y social (UNICEF, 2000). Su objetivo, dentro de los programas 
para las personas en conflicto con la ley, es gencrar cambios positivos. que permitan su reinserción social(Instituto Interamericano de Derechos Humanos: I.I.D.H.. 1983), además de proveer lineamientos básicos de escolarización.

Aprender es un proceso mental e individual, que se encuentra mediatizado por objetivos. contenidos o contextos culturales (Ausubel. 1983), e influido por las condiciones físicas y emocionales del individuo tales como, el uso o abuso de drogas, la historia de violencia física o emocional, el abandono afectivo, los vínculos familiares, el desuso en sus facultades mentales y su condición de privado de libertad. En él, la evaluación es vista en su dimensión sumativa y formativa y en el marco de una visión de proceso. El contexto está circunscrito tanto el espacio físico. como al cultural y emocional en el que diariamente conviven profesores. estudiantes y otros funcionarios ligados al quehacer educativo. Los recursos de aprendizaje son todos aquellos materiales -libros, revistas, vídeos u otros- que permiten aprender.

En todo proceso de aprendizaje participa una serie de sujetos que hacen posible el acto de aprender; uno de ellos es el profesor. Este es el responsable directo de organizar la experiencia de aprendizaje además de otras funciones inherentes a su cargo: realizar valoraciones de acompañamiento educativo dirigidas al juez que evaluará la permanencia o no del joven dentro del Centro; aplicar pruebas de diagnóstico para determinar entre otras cosas, el deterioro intelectual del muchacho, a raíz del uso de drogas u otros factores, para luego, ubicarlo en el nivel que le corresponde cursar. También, se incorpora a un trabajo integral, con las diversas áreas que tratan al menor: área de convivencia, capacitación y trabajo, atención a la violencia y la atención jurídica, además de otras funciones inherentes a su cargo. Actualmente, están nombradas dos maestras'y tres profesores por parte del Ministerio de Educación Pública, una profesora en el área de los Estudios Sociales y una directora, nombradas por parte del Ministerio de Justicia. En la investigación, se pidió la colaboración a cinco docentes, la directora del centro, a la encargada del área educativa y a dos miembros de las escuadras de seguridad.

Junto al docente, se encuentran los miembros del cuerpo de seguridad, cuya función es brindar protección en caso de violencia, (estos vienen a ser, los elementos de contención dentro del aula).

En el caso de los estudiantes, ellos son personas menores en proceso de formación, imaginativas y creativas, con habilidades que han sido aprendidas y olvidadas en contacto directo con su realidad. Una realidad que ha sido determinante 
en su proceso de aprendizaje y que ha estado mediatizada por múltiples factores que van desde el consumo de drogas a muy temprana edad, el desuso en las facultades mentales del joven, la deserción escolar o la indigencia. En cuanto a la población con la cual se trabajó, todos tienen la condición de sentenciados y están inscritos en el Programa de Educación Abierta del Instituto Costarricense de Educación Radiofónica, ICER. Ellos responden a los seudónimos de: "Cara Cortada", "René" y "Fut Fut".

La población penal juvenil en Costa Rica es la más reducida dentro de los centros de atenció; sin embargo, es la más necesitada. Debido a sus características, presenta mayores probabilidades de delinquir, pero también, de ser rescatada de su inclinación delictiva, (Rojas, 1996) por medio de la educación.

Ahora bien, el Paradigma sobre el cual está basada esta investigación es el Naturalista; es decir, pretende analizar y entender la realidad en su contexto natural, interpretar los fenómenos de acuerdo con los significados que estos tienen para los sujetos inmersos en esa realidad. El enfoque que caracteriza a este paradigma es de tipo cualitativo y etnográfico, lo que interesa es desentrañar los significados que tienen las acciones que realizan los sujetos que conforman el hecho educativo dentro del Centro de Formación Juvenil Zurquí, considerado en su dimensión holística, global e integral. En este caso, el criterio usado para validar los datos fue la triangulación. Para la recopilación de la información se usaron técnicas propias de la investigación cualitativa, tal es el caso de las Tablas de Observación, entrevistas dirigidas abiertas y en profundidad.

\section{La Confrontación de los hechos: El qué, el cómo y el para qué de la educación en el Centro de Formación Juvenil Zurquí}

\footnotetext{
"Somos igual que cualquiera; el único derecho que hemos perdido, es el de la libertad”. (Cara Cortada, 2001)
}

La educación dentro del Sistema Penitenciario tiene como objetivo brindar condiciones necesarias que faciliten la reinserción social del joven, que este construya un proyecto de vida más digno y gratificante, impulsarlos a la búsqueda de algo más que el encierro, las drogas, las pandillas, la deambulación y la violencia en cualquiera de sus formas, además de prevenir la reincidencia. Sin embargo, la falta de comprensión, apertura y credibilidad en el muchacho infractor y en su proceso de rehabilitación, le provoca desmotivación y estigma, 
lo cual da al traste con cualquier buena intención de cambio en el joven.. Tal y como lo demuestra el siguiente comentario hecho por René:

"Un domingo llegó una señora a visita, y me contaba que a su hijo lo tienen en una sección donde no están los asesinos -mencionó los nombres de ellos-; al decirle que yo era uno de ellos, la señora salió caminando rápido, se atarantó, no sabía para dónde coger, y a mí eso me dio un colerón, porque a nosotros nos ven como bichos raros" (René: 2000).

Las alternativas educativas a las que tiene acceso la población penal del Centro de Formación Juvenil Zurquí son de tipo andragógica, creada para adultos y dirigida hacia una población penal compuesta por jóvenes adolescentes y niños.

El espacio físico del centro es sumamente amplio: zonas verdes, canchas, oficinas, talleres, y las diversas secciones donde duermen los muchachos, entre otros. Las aulas -que son muy espaciosas- no tienen puertas, esto como medida de seguridad. Existen dentro del centro, seis secciones: una sección para los jóvenes de 12 a 15 años, la sección A para los de primer ingreso, la $\mathrm{B}$ para los reincidentes, la $\mathrm{C}$ para los sentenciados y la $\mathrm{D}$ para atender a los jóvenes con mayor trayectoria delictiva, y por ende, ocupan mayor contención. Esta se conoce con el nombre de "Bochos" (Equipo Técnico, Centro de Formación Juvenil Zurquí, 2000) .

La escuela alberga cincuenta muchachos -este número puede variar en cualquier momento- y su distribución depende de la especialidad, el ciclo de la educación, y las condiciones de ingreso del joven. Todos están adscritos dentro del Programa de Educación Abierta del Instituto Costarricense de Educación Radiofónica, ICER. Esta oferta educativa, concibe el aprendizaje como una experiencia concreta, vivencial y ligada a nuestras experiencias cotidianas, tal y como lo manifestó uno de los docentes:

"Cuando hablábamos del tema de desastres naturales, de la vegetación, René siempre me lo relaciona con su vida en Sixaola. Un día hablábamos del Amazonas, de sus bosques, el me decía que en Sixaola se ve todavía mucha vegetación, que aún se podía respirar aire puro; ahí hay mucha vegetación, en Talamanca también" (Docente 5; Entrevista en profundidad: 2000)

Dentro del planeamiento curricular se incluyen objetivos, contenidos. estrategias de aprendizaje. técnicas de evaluación y los recursos didácticos. 
De estos últimos, el más usado es el libro de texto del Instituto Costarricense de Enseñanza Radiofónica, tanto para Primaria, como para Secundaria. En cuanto a estos textos, los docentes apuntan que están desactualizados, que no traen mayores actividades, son poco profundos, poco directivos, su información es errónea y presenta definiciones ambiguas; sin embargo, sirven de guía al alumno y al profesor (Docente 3, Entrevista 1, 2000), ya que ahí vienen los contenidos que evalúa el Ministerio de Educación Pública, M.E.P., en los exámenes (Docente 4, Entrevista 1, 2000). Los textos usados son los de Educación Abierta: Térraba, Zapandí, y Ujarrás, llamados así para diferenciarlos del Sistema de Educación Formal. Estos se trabajan mediante guías, resúmenes, fichas o juegos didácticos.

El material escrito se combina con la utilización de audiovisuales: vídeos, películas, transparencias, diapositivas además del uso de mapas, pizarra, tiza y borrador. Su uso depende del tema y de la situación emocional del estudiante. Los docentes coincidieron en manifestar que la metodología que debe usarse para trabajar con este tipo de población, tiene que ser dinámica y participativa. Sin embargo, la metodología empleada, es conductual, en ella el profesor diseña las actividades, y los alumnos las desarrollan por sí mismos (Docente 1, Entrevista 12000 ). En cuanto a la evaluación, esta ha quedado relegada a la función meramente sumativa, es decir, a la aplicación de quices en forma oral o escrita y a una incipiente evaluación formativa que busca desarrollar buenos hábitos de limpieza e higiene en el muchacho.

\section{Los Sujetos Cognoscentes}

\section{Los Docentes}

Los docentes reciben diversos apelativos: "Facilitadores", "Niña", "Maestra", "Profesor (a)" o son llamados por su nombre de pila. Esto, según el nivel escolar y la edad del joven. Ellos trabajan con los estudiantes en forma grupal o individual. La motivación que brinda el docente es vital para desarrollar las actividades dentro del aula; esto es reconocido y valorado por los mismos jóvenes, a tal punto que provoca en ellos un cambio, un compromiso y una responsabilidad. Así lo manifestó Cara Cortada:

"Yo, mientras se revisa la lección, trato de dar lo mejor y cumplir con las tareas asignadas por la profesora y he mejorado mucho mi comportamiento con mis compañeros." (Cara Cortada: 2000) 
La forma de trabajo de un docente y su actitud ante los alumnos queda plasmada a partir del momento en que ingresa al aula, en sus ritos de iniciación, en las actividades que organiza, lo cual es percibido positiva o negativamente por el discente; así lo plantea Cara Cortada:

“...Ella siempre viene con una sonrisa en el rostro y pienso que eso no es normal con los demás profesores. A ella le gusta mucho su trabajo con nosotros, los menores privados de libertad, ella nos entiende (...).'(Cara Cortada: 2000)

El grado de significatividad de una materia depende del contenido mismo, de la utilidad práctica que este tenga y de los recursos que el docente emplee. El docente busca despertar en el educando algunos intereses de cambio como: dependencia por independencia, competencia por cooperación, aceptación por crítica, egocentrismo por solidaridad, individualismo por integración, conservadurismo por innovación y abstracción por experiencias vividas (Rodríguez, 1996)

\section{Los Guachimanes o agentes de seguridad}

El área de seguridad está conformada por dos escuadras, las cuales trabajan ocho días y descansan ocho días. A estas se las llaman "Guachimanes" o agentes de seguridad. En relación con su función uno de ellos manifestó que:

"Soy vigilante, tengo cuarenta años de edad y dieciocho de trabajar para el sistema penitenciario. Mi función dentro del área de seguridad en un centro como este, es la de velar por la integridad física de los privados de libertad, de las instalaciones, funcionarios administrativos, profesores y velar por el orden. (Vigilante 1: 2000)

También se ocupan de resguardar los bienes materiales del centro, requisar a los internos, castigar las faltas que el muchacho cometa contra un docente u otro interno. El nexo entre estos y el área educativa es fundamental, ya que los miembros del cuerpo de seguridad son los encargados de traer a clases a los estudiantes, vigilar desde fuera, requisar al alumno una vez que las clases terminan (para evitar el intercambio de drogas), esposarlos nuevamente y llevarlos a sus respectivas secciones. No obstante, y como en cualquier centro penal, su labor es sumamente riesgosa. Uno de los vigilantes nos relató al respecto su experiencia: 
"El peligro es latente. La situación más riesgosa que he enfrentado fue el secuestro que hicieron menores a un funcionario, él era un psicólogo. Fue planificado por dos menores. Estábamos en el Centro Juvenil San José, de esto hace dos años. Fue una situación riesgosa y delicada. Al Psicólogo lo agarraron y lo amordazaron. Ellos cerraron el módulo, lo agredieron, golpearon y ellos decían que si no abrían lo apuñalaban. Sacaron a los vigilantes. Los portones estaban cerrados con candados. Pidieron un carro para fugarse. Al final tuvimos que intervenir". (Vigilante 1: 2000)

\section{Aquellos que han perdido su libertad: "Cara Cortada", "René" y "Fut Fut"}

"La ambición y la droga fueron la causa principal para destruir una familia que la cáreel separó. Por vender sustancias prohibidas un hombre y una mujer la cárcel fueron a visitar; el señor, antes de partir, le habló a su hijo diciéndole que tomara su puesto; en ese momento para ese joven comenzaría una nueva vida....." René Carta personal, 2001

La institución alberga jóvenes infractores, mujeres y hombres, quienes presentan ciertas particularidades en común: han sido producto de un contexto sociocultural y están influidos por las normas culturales presentes en ese ambiente; sus vínculos familiares son pobres o nulos: tienen actitudes negativas de valoración personal y social, además de formas específicas de violencia, las cuales repercuten en el proceso de socialización del menor (Mora, 2000).

La misma autora afirma (Mora, 2000) que si bien es cierto no se puede ser determinista a la hora de construir un perfil del menor infractor, aquellos que son detenidos, detectados y procesados presentan ciertos rasgos similares. En su mayoría, provienen de zonas marginales, rurales y urbanas tales como, "Las Latas" en Paraíso, Quircot, Cieneguita, Roosevelt, Sixaola, Limoncito o Rockefeller, Los Guido, Los Cuadros, León Trece, Rincón Grande de Pavas, Guararí, Fray Casiano o Barranca en Puntarenas, Nazareth en Liberia, entre otros. (Docente 5: Entrevista en profundidad: 2001). Por lo tanto, su mundo social, comunal y familiar está marcado por la pobreza, la violencia, el abandono afectivo, el consumo de drogas. problemas con la justicia y la falta de oportunidades.

Lo anterior no significa (Mora, 2000) que no hayan tenido afecto, apoyo y experiencias positivas durante su desarrollo, pero el crecer dentro de grupos de referencia violentos y estigmatizados, incidió de alguna manera en ellos. Muchos de estos jóvenes han sido abandonados por uno de sus progenitores o por 
ambos, han deambulado por las calles, han estado en albergues del Patronato Nacional de la Infancia o en otros particulares, tales como: "La ciudad de los Niños", "Amor en la Calle", o "Roblealto", entre otros (Docente 5; Entrevista en profundidad: 2000). Les faltan límites de contención, son desertores de la educación, utilizan armas y además, presentan una desconexión moral hacia el delito, al depositar la culpa en los demás. Un ejemplo de ello lo dio "Cara Cortada", durante una lección de Estudios Sociales:

“A mi no me interesa saber del Estado Oligárquico, ni el juez me lo va a preguntar: lo único que me interesa saber es cómo le voy a dar a la persona para que se muera de inmediato, dónde le voy a dar, en ese lugar específico" (Cara Cortada: 2000).

Su situación se agrava cuando incorporan a su cotidianidad el consumo de drogas. El círculo de violencia se cierra, y solo quedan las marcas en forma de cicatrices en sus espaldas o piernas como producto de cuerdas, alambres o cuchillas.

Los conflictos propios de la adolescencia hacen que su situación escolar y legal sea más crítica, ya que su mundo interno ha sufrido una fuerte modificación, asociado a los cambios físicos propios de la etapa en la que se encuentra, cambios sociales, a nivel de pensamiento y en las relaciones interpersonales; por ende, su percepción del mundo es muy distinta a la de otros jóvenes.

Además de sus carencias afectivas, han construido patrones y valores particulares, que se salen de las reglas socialmente establecidas, sin medir las consecuencias de sus actos, y por éstos, son detectados y detenidos, al pasar a formar parte de la categoría de "DELINCUENTES JUVENILES" (lo cursivo es nuestro) (Mora, 2000). El cuadro No. 1 muestra algunos datos generales de los estudiantes con quienes se trabajó durante la investigación.

\section{Cuadro No. 1}

\section{Datos Generales de los informantes-estudiantes}

\begin{tabular}{|l|l|l|l|}
\hline Seudónimo & Cara Cortada & Fut - Fut & René \\
\hline Sexo & Masculino & Masculino & Masculino \\
\hline Edad & 15 & 17 & 16 \\
\hline Nacionalidad & Costarricense & Costarricense & Costarricense \\
\hline $\begin{array}{l}\text { Razón por la que se } \\
\text { encuentra en el centro }\end{array}$ & $\begin{array}{l}\text { Tentativa de Homicidio } \\
\text { calificado y Robo } \\
\text { agravado }\end{array}$ & Robo agravado & $\begin{array}{l}\text { Homicidio y Robo } \\
\text { agravado }\end{array}$ \\
\hline
\end{tabular}




\section{Cuadro No. 1}

Datos Generales de los informantes-estudiantes

\begin{tabular}{|l|l|l|l|}
\hline Condición Judicial & Sentenciado & Sentenciado & Sentenciado \\
\hline Nivel que cursa & Sétimo & Sétimo & Sétimo \\
\hline $\begin{array}{l}\text { Razón por la que dejó de } \\
\text { estudiar }\end{array}$ & Legal & Perezoso y vago & Por vago \\
\hline Consumo de drogas & $\begin{array}{l}\text { Alcohol desde los 13 } \\
\text { años }\end{array}$ & $\begin{array}{l}\text { Marihuana desde los 15 } \\
\text { años }\end{array}$ & No \\
\hline Apoyo Familiar & $\begin{array}{l}\text { Sí, antes y después de } \\
\text { legar al centro }\end{array}$ & Ibid & Ibid \\
\hline $\begin{array}{l}\text { Propósito al recibir } \\
\text { lecciones }\end{array}$ & $\begin{array}{l}\text { Seguir adelante. porque } \\
\text { me quedé atrasado y } \\
\text { porque creo que es algo } \\
\text { que tengo que saber para } \\
\text { uso en mi vida. }\end{array}$ & $\begin{array}{l}\text { Salir adelante con mis } \\
\text { metas. }\end{array}$ & $\begin{array}{l}\text { Aprender más. continuar } \\
\text { con mis estudios, ser útil } \\
\text { a la sociedad. }\end{array}$ \\
\hline $\begin{array}{l}\text { Expectativas } \\
\text { Seguir mis estudios. } \\
\text { Llegar a ser alguien en } \\
\text { la vida y no un } \\
\text { delincuente. }\end{array}$ & $\begin{array}{l}\text { Seguir estudiando. Salir } \\
\text { a trabajar y ser mejor en } \\
\text { la vida. }\end{array}$ & Seguir estudiando. \\
\hline
\end{tabular}

Fuente: Entrevista. Heredia 2000

De él se deduce, que pese a su proceso de prisionalización, estos jóvenes tienen expectativas, proyectos y deseos de cambio, los que deben ser canalizados positivamente, con la ayuda de docentes y administrativos del centro; con ello se cumple, tanto con las disposiciones legales, como con el deber moral y social, que tiene todo adulto que trabaje en educación con jóvenes menores de edad.

\section{Los resultados de esta investigación}

En cuanto a las conclusiones más importantes de esta investigación, se partió de lo más general a lo más específico, del concepto de educación que se maneja en el centro, hasta las características que poseen los sujetos congnoscentes, considerados como elementos fundamentales en el proceso de aprendizaje. La educación que se imparte en el centro es de tipo andragógica, creada para una población de adultos, por tanto, no contempla ni las características, ni las necesidades de los muchachos que ahí se encuentran. Sin embargo, la educación que se ofrece lleva un doble propósito: cumplir las disposiciones jurídicas nacionales e internacionales en materia de educación y servir de vía para lograr un cambio positivo en el joven y en su reinserción social. 
Por ende, es una educación formativa, aunque poco consistente debido a la falta de claridad en materia educativa.

La poca motivación y las condiciones difíciles de aprendizaje, implican una lucha diaria contra el desinterés, el desuso en las facultades mentales del muchacho y el síndrome de abstinencia entre otros. Aunque el espacio físico del centro es muy amplio e idóneo, el espacio emocional está mediatizäo por situaciones especiales, las cuales generan una agresión verbal constante en contra del docente, agresiones que van desde la apatía, las frases coercitivas, los gestos inapropiados, hasta la masturbación en el aula.

El Programa Educativo que se imparte es el del Instituto Costarricense de Educación Radiofónica. El docente es un facilitador, la metodología que propone es participativa y la evaluación es unidireccional y de tipo sumativa. Este se desarrolla en el centro, bajo un enfoque academicista, tradicional, bancario y conductual. El profesor complementa su labor con recursos tales como, audiovisuales, textos, rotafolios, filminas y láminas educativas además de juegos.

Es evidente la falta de apoyo institucional por parte del Ministerio de Justicia y del Ministerio de Educación, ya que no se han diseñado los mecanismos necesarios que evalúen la experiencia en el centro. El personal docente está integrado en su mayoría por profesionales graduados, aunque con poca experiencia dentro del Sistema de Educación Abierta y con este tipo de población. De ahí que predomine en ellos un sentimiento de frustración y desmotivación.

De acuerdo con la población estudiada, es válido afirmar que estos son seres humanos pasivos y receptivos, ya que la transferencia de conocimientos es de carácter lineal. Estos jóvenes poseen ciertas características comunes: en su mayoría provienen de zonas de atención prioritaria; han tenido contacto temprano con la calle, el sexo y las drogas; sus vínculos familiares son escasos, nulos o de una calidad muy pobre; tienen severos problemas de aprendizaje, falta de hábitos de estudio, inseguridad emocional; han sido desertore's de la educación; han sufrido abandono; tienen formas abusivas de relacionarse; son hiperactivos o pasivos y con dificultad para solucionar sus problemas interpersonales.

A pesar de lo anterior, son muchachos que tienen sueños, ilusiones, esperanzas, expectativas de vida, deseos de superación, necesidades afectivas y que necesitan una motivación constante que les impulse a desarrollar esa capacidad intelectual que, como seres humanos, poseen.

\section{Las recomendaciones finales}

Entre las recomendaciones que se plantearon está la necesidad de analizar en forma exhaustiva el Programa de Estudio que se aplica en el centro, los contenidos y la extensión de estos. Evaluar la posibilidad de diseñar un currículum 
que contemple los contenidos de la educación formal, pero que responda a las particularidades de los muchachos. Redefinir el concepto de educación para adolescentes, considerando al joven en su dimensión como persona. con una visión de mundo diferente a la del adulto y, por ende, el estudiante deberá ser concebido como elemento y fuente del currículum.

Las estrategias de aprendizaje tienen que considerar las características biológicas, psicológicas, socioculturales y la situación jurídica del muchacho. Lograr una coordinación más eficiente entre los Ministerios de Justicia y Educación Pública, a fin de que se agilicen trámites para la inscripción y presentación de pruebas, así como para la entrega de los títulos. Además, que los exámenes presenten las respectivas modificaciones de acuerdo con los problemas de aprendizaje que se detecten en los muchachos. Asimismo, que se brinden asesorías sobre el sistema de estudio del ICER: la evaluación, las técnicas didácticas, la adecuación curricular y los problemas de aprendizaje.

Hacer una evaluación periódica sobre el proceso educativo que se desarrolla en el centro, mediante reuniones entre el personal docente y administrativo y entre las diferentes personas, que integran las áreas que trabajan con los muchachos. Coordinar esfuerzos y acciones para que el currículum que se aplique en el centro considere también las necesidades laborales de los jóvenes y su inserción en el mercado laboral. Para ello se debe brindar talleres de capacitación en diversas áreas y especialidades. Además, que se consideren ciertos criterios técnicos a la hora de nombrar al personal docente tales como; perfiles, áreas de especialización, experiencia en trabajo con personas menores de edad infractoras y bajo el Sistema de Educación Abierta, entre otras.

\section{Referencias}

\section{Fuentes primarias}

DIRECTORA 1. Entrevista. Heredia, 2000

DIRECTORA 2. Entrevista. Heredia, 2000.

CARA CORTADA. Entrevista personal a profundidad. Heredia, 2000.

CARA CORTADA. Observación. Heredia, 2000

DOCENTE 1. Entrevista 1. Heredia, 2000

DOCENTE 2. Entrevista 1. Heredia, 2000

DOCENTE 3. Entrevista 1. Heredia, 2000

DOCENTE 4. Entrevista 1. Heredia, 2000

DOCENTE 5. Entrevista en Profundidad. Heredia, 2000

DOCENTE 5. Entrevista en Profundidad. Heredia, 2001

DOCENTE 1. Entrevista 2. Heredia, 2000 
DOCENTE 2. Entrevista 2, Heredia, 2000

DOCENTE 3. Entrevista 2, Heredia 2000

FUT-FUT. Entrevista personal a profundidad. Heredia, 2000

FUT-FUT. Observación. Heredia, 2000.

PSICÓLOGA. Entrevista. Heredia 2001.

RENE. Entrevista personal a profundidad. Heredia, 2000.

RENÉ. Observación. Heredia, 2000.

RENÉ. Carta. Heredia, 2001

VIGILANTE 1. Entrevista. Heredia, 2000

VIGILANTE 2. Entrevista. Heredia, 2000

\section{Fuentes secundarias}

Ausubel, David y otros. Psicología Educativa: Un punto de vista cognitivo. Segunda Edición: Editorial Trillas, México, 1983.

Constitución Política. Publicaciones Jurídicas, San José, 1995.

Costa Rica. Ministerio de Justicia y Gracia. Equipo Técnico del Centro de Formación Juvenil Zurquí. La intervención especializada en el Centro Juvenil Zurquí a Población identificada con características de difícil manejo "Sección D Mixta". Heredia, 2000.

Instituto Costarricense De Enseñanza Radiofónica. Aprendamos a estudiar con "El maestro en casa: una guía para jóvenes y adultos que ingresan a la Educación Abierta. San José: I.C.E.R., 2000.

Mora, D. Ada Luz. Dirección Nacional y Atención a la Población Penal Juvenil. San José: Ministerio de Justicia y Gracia, Junio 2000.

Rodríguez León, Edgar. Plan de atención técnica correspondiente al área de servicios educativos. San José, 1992.

Rojas, Norberto. Lineamientos para el mejoramiento de los servicios educativos del Centro de Orientación Juvenil Luis Felipe González Flores. Tesis de graduación para optar el grado de Licenciatura en Ciencias de la Educación con énfasis en Administración Educativa: Universidad de Costa Rica, San José, 1996.

UNICEF. Ley de Justicia Penal Juvenil de Costa Rica. San José: UNICEF, 2000. 